\title{
A mixed-methods review of how quality improvement is represent in the UK postgraduate medical curricula
}

\author{
Author: Rebecca Smith ${ }^{\mathrm{A}}$
}

\begin{abstract}
Introduction
Today's medical curricula shape the institutional culture of tomorrow. There is an opportunity within the medical curricula to embed improvement methodology at the heart of practice, and ignite meaningful change throughout the health service. Therefore, it is imperative that quality improvement (QI) is well-characterised in the medical curricula.
\end{abstract}

\section{Aim}

To determine how QI is represented in postgraduate medical curricula.

\section{Method}

Sixty postgraduates' medical curricula and decision aides were reviewed. Text related to audit or quality improvement was extracted and coded for thematic analysis. QI engagement assessment measures were also reviewed.

\section{Results}

There is a significant variation between training programmes with regard to the number of audits or QI projects expected of trainees.

The text of the curricula was broadly congruent. Common themes included: the PDSA cycle, involvement of the multidisciplinary team and clinical governance.

\section{Conclusion}

QI should be pervasive across all healthcare settings. Despite this, there is considerable variation in the requirements for junior doctors in different training programmes. This suggests a lack of consensus on how many projects constitute an appropriate level of experience. Therefore, it is worth considering if there are potentially more meaningful methods of ensuring exposure to QI methodology.

Many curricula require a progression from participation in QI, in formative years, to leading a project and then simply supervising. This hierarchical view does follow a logical progression, but risks ingraining the damaging assumptions that a senior doctor's role in QI should be passive. Similarly, this progression encourages trainees to pursue leadership of small projects with limited impact, rather than become a participant in larger scale projects that are more likely to deliver change.

These curricula bear the hallmarks of an assessment methodology that needs to be administered in a standardised fashion, efficiently, to a large population. These assessment metrics have led trainees to realign their perception of QI, and temper their ambitions for change. As a result, only a minority of trainee interventions have a permanent positive impact, despite significant amounts of time being invested by trainees.

\section{Recommendations for curriculum redesign}

$>$ Remove the arbitrary requirement for engagement with a certain number of projects and allow a wider range of evidence for engagement, such as: conference attendance, online education and involvement with ongoing iterative projects.

> Remove hierarchical terms from the QI curriculum.

> Consider whether cross-specialty collaboration is required in the setting of assessment metrics, to reduce the variability between training programmes.

\section{Conflicts of interest}

None declared.

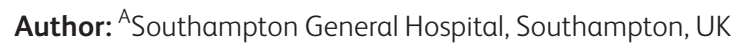

